\title{
Caractéristiques physico-chimiques des eaux souterraines du socle du bassin versant de Goroubi dans la commune de Torodi /Liptako nigérien
}

\author{
Samaila Djoumaye DJAHADI ${ }^{*}$, Issoufou SANDAO ${ }^{1,2}$, Yacouba AHMED $^{1}$, \\ Moussa HAROUNA et Boureima OUSMANE ${ }^{1}$ \\ ${ }^{1}$ Université Abdou Moumouni de Niamey, Faculté des Sciences et Techniques, Département de Géologie ; \\ $B P$ : 10662 Niamey, Niger. \\ ${ }^{2}$ Ministère de l'Hydraulique et de l'Assainissement, BP : 517 Niamey, Niger. \\ *Auteur correspondant ; E-mail : ddjoumeye@yahoo.fr
}

\begin{tabular}{lll}
\hline Received: 16-07-2021 & Accepted: 27-11-2021 & Published: $31-12-2021$ \\
\hline
\end{tabular}

\section{RESUME}

La région d'étude est située en zone de socle dans la commune rurale de Torodi, partie sud-ouest du Niger, où l'alimentation en eau des populations est faite à partir des eaux de surfaces et des nappes superficielles. Suite à la vulnérabilité de ces dernières, liée à la sècheresse et à la pollution, une attention plus particulière a été accordée aux eaux des nappes plus profondes à travers le fonçage des puits modernes et des forages. Cependant, des études récentes réalisées sur différents points d'eau situés en d'autres zones de socle, ont révélé d'importantes valeurs en éléments chimiques indésirables parfois même supérieures aux normes OMS. Evaluer la qualité des eaux de cette région par les analyses physico-chimiques s'avère aussi importante surtout dans ce contexte de pollution grandissante et pour une gestion rationnelle des ressources. L'approche méthodologique a consisté aux prélèvements de dix échantillons d'eau des puits et des forages pendant le mois de Mai pour les analyses physicochimiques ( $\mathrm{HCO}^{-}, \mathrm{Mg}^{2+}, \mathrm{Na}^{+}, \mathrm{K}^{+}, \mathrm{Ca}^{2+}, \mathrm{NO}_{3}^{-}, \mathrm{Fet}$ ), à leur étude statistique à travers l'Analyse en Composante Principale et au calcul de l'indice de saturation. Les résultats ont montré que certains points d'eau ne sont pas conseillés à la boisson pour causes des teneurs en Nitrates et en Fet dépassant les normes. Ces eaux étaient faiblement minéralisées, sous saturées à la calcite, à la dolomite et présentaient deux faciès chimiques : le faciès de type bicarbonaté calcique et magnésien et bicarbonaté sodique et potassique. La méthode statistique de l'Analyse en Composante Principale ACP a mis en évidence l'hydrolyse des silicates en présence du $\mathrm{CO}_{2} \mathrm{du} \mathrm{sol}$. (C) 2021 International Formulae Group. All rights reserved.

Mots clés : Aquifères discontinus, qualité des eaux, ACP, hydrolyse, bassin versant de Goroubi, Niger.

\section{Physico-chemicals characteristics of the watershed Goroubi's groundwater in Commune of Torodi/Liptako of Niger}

\begin{abstract}
The study area is located to the basement of rural commune of Torodi, southwest of Niger, where population water supplied is made from surface water and superficial aquifers. With the vulnerability of these latter, due to drought and pollution, more attention has been paid to deep aquifers by modern wells and boreholes
\end{abstract}


drilling. However, recent studies made on different water points located on other basement areas, revealed important values of undesirable chemical elements that exceed the World Organization Health(WHO) drinking water guidelines. In context of increasing pollution and management of resources, evaluate the quality of these waters is important. Ten samples of water are collected from wells and boreholes during month of May 2018 and analyze from major and trace elements ( $\mathrm{HCO}^{-}, \mathrm{Mg}^{2+}, \mathrm{Na}^{+}, \mathrm{K}^{+}, \mathrm{Ca}^{2+}, \mathrm{NO}_{3}{ }^{-}$and Fet). A principal Component Analyses PCA and the saturation index are also used. Results of analyses shown that some water points are not recommended for drinking causes of high levels of Nitrates and iron total exceeding guidelines of the WHO. Waters are weakly mineralized, undersaturated from calcite and dolomite and shown two types facies: bicarbonate calcic and magnesic and bicarbonate sodic and potassic. The statistical method of ACP highlights the hydrolysis of silicates in the presence of soil $\mathrm{CO}_{2}$.

(C) 2021 International Formulae Group. All rights reserved.

Keywords: Discontinuous aquifers, water quality, ACP, hydrolysis, Goroubi watershed, Niger.

\section{INTRODUCTION}

Les eaux souterraines constituent la principale source d'approvisionnement pour la majorité des populations nigériennes, surtout rurales. Cependant, ces ressources sont souvent très vulnérables aux bouleversements climatiques et leur qualité physico-chimique est de plus en plus menacée par les actions anthropiques à travers l'utilisation abusive d'engrais pour les besoins de l'agriculture et les cultures irriguées d'une part, et par les rejets industriels et ménagers d'autre part. La région du moyen bassin versant de Goroubi, objet de notre étude, occupe la commune rurale de Torodi située au Sud-ouest du Niger. Dans ces zones, les réserves d'eaux souterraines sont localisées dans les couloirs préférentiels des fractures et dans les couches superficielles des alluvions souvent vulnérables aux longues saisons sèches et responsables de nombreuses maladies hydriques. Pour protéger la santé des populations, l'Etat du Niger, soutenu par les partenaires au développement a procédé à la réalisation de plusieurs points d'eau modernes captant les aquifères discontinus du socle fissuré et altéré censés fournir des eaux potables et pérennes. Cependant, des études réalisées dans le but d'évaluer la qualité des ressources en eau des nappes du socle, ont montré une pollution par les éléments chimiques tels que le Fluor, les Sulfates, les Nitrates et les Nitrites. C'est le cas des études faites dans la commune de Téra (Ousmane et al., 2010 ; Babayé et al., 2016) et dans la région de Zinder (Souley et al., 2018; Sandao et al., 2019). Ces teneurs anormalement élevées dont l'origine est naturelle notamment liée à la lithologie des réservoirs aquifères dans certaines zones (Sandao et al., 2019), est cependant dans d'autres régions de nature anthropique (Favreau et al., 2004 ; Abdou Babayé et al., 2016). Ces fortes teneurs ont pour corollaire la persistance des maladies hydriques, l'abandon des ouvrages en raison des mauvaises propriétés organoleptiques et par conséquent la baisse du taux de desserte en eau, annihilant ainsi les efforts déployés par l'Etat.

Dans un contexte de pollution grandissante et pour une gestion rationnelle et efficiente des ressources en eau, surtout pour l'une des régions les moins desservies ayant un taux de desserte en eau potable inférieur à $40 \%$ (PSeau, 2018), la détermination de la qualité physico-chimique des eaux souterraines du moyen bassin versant de Goroubi afin de connaitre leur aptitude à la consommation humaine s'avère nécessaire. De façon plus spécifique, il s'agissait de sélectionner dix points d'eau représentatifs de la zone qui ont été échantillonnés et analysés, puis traiter les résultats à base des logiciels adéquats (Canvas, Statistica, diagramme, ArcGis) afin de déterminer leurs caractéristiques physicochimiques et les mécanismes à l'origine de leur minéralisation.

\section{MATERIEL ET METHODES}

\section{Présentation de la zone d'étude}

Le moyen bassin versant du Goroubi s'étend sur une superficie d'environ $2207 \mathrm{~km}^{2}$ et s'inscrit entre $13^{\circ}$ et $13^{\circ} 32^{\prime}$ de latitude Nord et entre $1^{\circ} 12^{\prime}$ et $1^{\circ} 58^{\prime}$ de longitude Est. Il est 
localisé dans la commune de Torodi appartenant au département de même nom, faisant partie de la région de Tillabéri localisée à l'Ouest du pays (Figure 1). Le climat de la zone est de type sahélo-soudanien avec deux saisons très contrastées : l'une plus longue et sèche d'octobre à mai, l'autre plus courte de juin à septembre est humide. Pendant la saison sèche, les journées et les matinées de décembre à janvier sont relativement fraiches ; par contre de mars à avril les températures sont élevées aussi bien de nuit que de jour.

Le cumul pluviométrique annuel varie de 200 à $900 \mathrm{~mm}$ sur la période allant de 1963 à 2020, au poste pluviométrique de Torodi. Cependant, la pluviométrie est inégalement répartie dans le temps et dans l'espace au cours d'une même saison et d'une saison à l'autre. Elle est à la base de la recharge des différents aquifères (aquifères discontinus du socle et alluviaux) assurant l'alimentation en eau des populations. Les valeurs de la température atmosphérique varient de $16^{\circ} \mathrm{C}$ en janvier à $41^{\circ} \mathrm{C}$ en Avril. Quant à l'évapotranspiration potentielle moyenne annuelle, elle peut dépasser les $2500 \mathrm{~mm}$ et reste supérieure aux précipitations sauf pendant les mois de Juillet et Août.

$\mathrm{Du}$ point de vue hydrographique, la région est constituée par la rivière du Goroubi et la Sirba qui compte plusieurs affluents dont entre autre le Lomona, le Silenké, le Mago et le Diguibari qui est le plus important. Ce réseau hydrographique compte également de nombreuses mares temporaires qui s'assèchent en saison sèche.

\section{Contexte géologique et hydrogéologique}

Sur le plan géologique, la région du moyen bassin versant de Goroubi appartient au socle paléoprotérozoïque de la dorsale de Man. Ces formations se répartissent en ceintures de roches vertes en alternances avec des plutons granitoïdiques. C'est précisément sur le pluton de Torodi que repose la zone d'étude. La lithologie des ceintures des roches vertes est formée essentiellement des roches métamorphiques notamment les metabasites, les métas diorites, méta andésite à composition minéralogique et structure variées (Babayé, 2012). Quant aux formations granitoïdiques, elles sont localement constituées de diorites quartziques à enclaves de restites biotitiques avec à certains endroits des granites à phénocristaux d'épidotes, des granites monzonitiques et calco-alcalins avec des injections de filons de quartz, ou de pegmatites. Ces différentes formations sont traversées par plusieurs failles cassantes multidirectionnelles (Figure 2).

La région comprend essentiellement deux types d'aquifères qui sont :

* L'aquifère superficiel dont le réservoir est composé par les alluvions et les colluvions ainsi que les altérites développées sur le socle. Il est à nappe libre et semble jouer un rôle capacitif. Les alluvions sont constituées pour la plupart des formations sablo-argileuses ; et est le souvent capté par des puits

* L'aquifère profond dont le réservoir est constitué par le socle cristallophyllien fracturé et fissuré. Cet aquifère est discontinu sur le plan hydraulique, pouvant exister seul ou sous-jacent à l'aquifère superficiel, jouant ainsi un rôle de drain. Cet aquifère profond est capté par des forages tandis que l'aquifère superficiel est capté par des puits et/ou des puisards.

Le matériel utilisé dans cette étude comprenait les outils et les données. Les données comprenaient entre autres les fiches techniques des forages, les documents, les cartes (géologique du Liptako et topographique feuille de Gothèye) et les données des paramètres physico-chimiques mesurés in situ et celles issus des analyses de laboratoires effectuées; à la Direction Régionale de l'Hydraulique et de l'Assainissement de Tillabéri. Les outils comprenaient quant à eux les équipements de terrain(le conductimètre, le $\mathrm{pH}$ mètre et le thermomètre) pour la mesure de la Conductivité électrique CE $(\mu \mathrm{S} / \mathrm{cm})$ de la Température $\left({ }^{\circ} \mathrm{C}\right)$ et du $\mathrm{pH}$ et les outils de laboratoire composés de grands appareils pour les analyses.

\section{Méthodologie}

\section{Choix des sites de et protocole d'échantillonnage}

La sélection des points à prélever a été basée sur une bonne distribution spatiale des 
points d'eau dans la zone, et a permis de prendre dix échantillons représentatifs sur les vingt-sept échantillons prélevés en Mai 2018. Ces dix échantillons ont concerné les eaux de huit forages et deux puits. Quant au prélèvement, il a été effectué dans des flacons en polyéthylènes de $500 \mathrm{ml}$ directement au robinet des forages. Par contre pour les puits, une puisette a été utilisée à cet effet. Avant le remplissage des flacons, ces derniers ont été rincés deux à trois fois et une certaine quantité d'eau a été prélevée pour la mesure des paramètres physiques $\left(\mathrm{T}^{\circ}, \mathrm{CE}, \mathrm{pH}\right)$. Les flacons, une fois remplis ont été hermétiquement fermés et étiquetés, puis mis dans une thermos pour permettre leur transport au laboratoire dans des meilleures conditions.

\section{Techniques analytiques des paramètres chimiques}

Les techniques analytiques utilisées étaient :

- La volumétrie pour la détermination des ions majeurs comme le calcium $\mathrm{Ca}^{2+}$, le magnésium $\mathrm{Mg}^{2+}$, les hydrogénocarbonates $\mathrm{HCO}_{3}^{-}$et les chlorures $\mathrm{Cl}$;

- La spectrophotométrie pour les ions ferriques $\mathrm{Fe}^{2+}$, sulfates $\mathrm{SO}_{4}{ }^{2-}$, fluorures $\mathrm{F}^{-}$, nitrates $\mathrm{NO}_{3}{ }^{-}$et nitrites $\mathrm{NO}_{2}{ }^{2-}$;

- La photométrie à flamme pour le dosage des ions sodium $\mathrm{Na}^{+}$et potassium $\mathrm{K}^{+}$.

\section{Traitement des données}

Le traitement a consisté au calcul des valeurs moyennes et des écart-types des différents paramètres ; à la comparaison des valeurs des paramètres entre elles et également celle des valeurs moyennes aux normes OMS (2011). Pour les paramètres chimiques, il a été déterminé la balance ionique afin de vérifier la fiabilité des analyses chimiques

Elle a été déterminée pour chaque échantillon à partir de la formule suivante :
$\mathbf{B i}=\frac{\left(\sum \% \text { Cations }-\sum \% \text { Anions }\right)}{\left(\sum \% \text { Cations }+\sum \% \text { Anions }\right)} * \mathbf{1 0 0}$

Avec, $\mathrm{Bi}$ : balance ionique exprimée, en $\%$ et les concentrations des cations et anions en méq/L. L'analyse est considérée acceptable lorsque les valeurs de $\mathrm{Bi} \leq \pm 6$

Les concentrations en éléments chimiques ont fait l'objet des différentes représentations graphiques (Diagramme de Piper et Shoeler Berkaloff), afin de connaitre les hydrofaciès des différents aquifères de la zone. Il a également été calculé les indices de saturation et d'étude statistique basée sur l'Analyse en Composantes Principales ACP afin de comprendre le processus de minéralisation des aquifères.

Représentations graphiques

Le diagramme de Piper est composé de deux triangles à la base dont un à gauche pour les pourcentages des concentrations des cations et celui de la droite réservé aux pourcentages des concentrations des anions. Au-dessus de ces deux triangles se trouve un losange, résumant le faciès chimique correspondant, regroupant les anions et les cations.

Quant au diagramme de Schoeler Berkaloff, il permet surtout de déterminer l'appartenance à une même famille ou un même processus de minéralisation. Sont portées sur ce type de diagramme, les concentrations des cations et anions majeurs en mg/L ou en méq/L.

\section{La dureté hydrotimétrique de l'eau}

La dureté est un indicateur du niveau des alcalino-terreux $\left(\mathrm{Ca}^{2+}\right.$ et $\left.\mathrm{Mg}^{2+}\right)$ dans l'eau. Elle traduit la capacité de l'eau à réagir avec le savon (Hassane Saley et al., 2019) et s'exprime en ppm de carbonate de calcium $\left(\mathrm{CaCO}_{3}\right)$ ou en ${ }^{\circ} \mathrm{f}$ (degré français) par l'équation suivante :

${ }^{\circ}$ th $=\mathrm{Ca}^{2+} * \frac{\mathrm{CaCO}_{3}}{\mathrm{Ca}}+\mathrm{Mg}^{2+} * \frac{\mathrm{CaCO}_{3}}{\mathrm{Ca}}$

Où :

${ }^{\circ}$ th $=$ titre hydrotimétrique, en ${ }^{\circ} \mathrm{f}$ ou en ppm de $\mathrm{CaCO}_{3}$ avec $1^{\circ} \mathrm{f}=10 \mathrm{mg} / \mathrm{L}$ de $\mathrm{CaCO}_{3}$

$\mathrm{Ca}, \mathrm{Mg}, \mathrm{C}$ et $\mathrm{O}$ : masses molaires exprimées en $\mathrm{g} / \mathrm{mol}$

$\mathrm{Ca}^{2+}$ et $\mathrm{Mg}^{2+}$ : concentrations exprimées en $\mathrm{mg} / \mathrm{L}$ 


\section{L'indice de saturation}

Il permet de déterminer la saturation à l'origine de la minéralisation des eaux souterraines vis-à-vis de certains minéraux tels que la calcite, le gypse et la dolomite. Il indique donc la capacité de dissolution ou de précipitation des minéraux dans l'eau et est défini par :

$I S=\log \left(\frac{P A I}{K_{S}}\right) \quad($ Yao, 2009)

Avec:

PAI : le rapport d'activité ionique

Ks : le produit de solubilité ; Ainsi si :

IS $<0$ : la solution est sous-saturée par rapport au minéral considéré

IS $>0$ : la solution est sursaturée au minéral avec une tendance à la précipitation

IS $=0$ la solution est en équilibre avec le minéral donc $\mathrm{PAI}=\mathrm{Ks}$
Cependant compte tenu des erreurs analytiques et de mesures du $\mathrm{pH}$ se traduisant dans le calcul de IS, la convention suivante peut être adoptée (Ousmane, 1988 ; Yao, 2009):

IS < - 0,5 la solution est sous-saturée, $-0,5<$ IS $<+0,5$ la solution est saturée et IS $>+0,5$ la solution est à la sursaturation. Dans la présente étude, les indices de saturation ont été automatiquement calculés à partir du logiciel Diagrammes grâce à la fonctionnalité Phreeqc.

\section{Analyse en Composantes Principales ACP}

L'ACP a pour but d'étudier d'éventuelles relations existant entre différents paramètres physico-chimiques. Dans cette étude, il a été utilisé douze variables afin de déterminer leur influence dans la minéralisation. L'ACP a été réalisé à l'aide du Logiciel Statistica7.1.

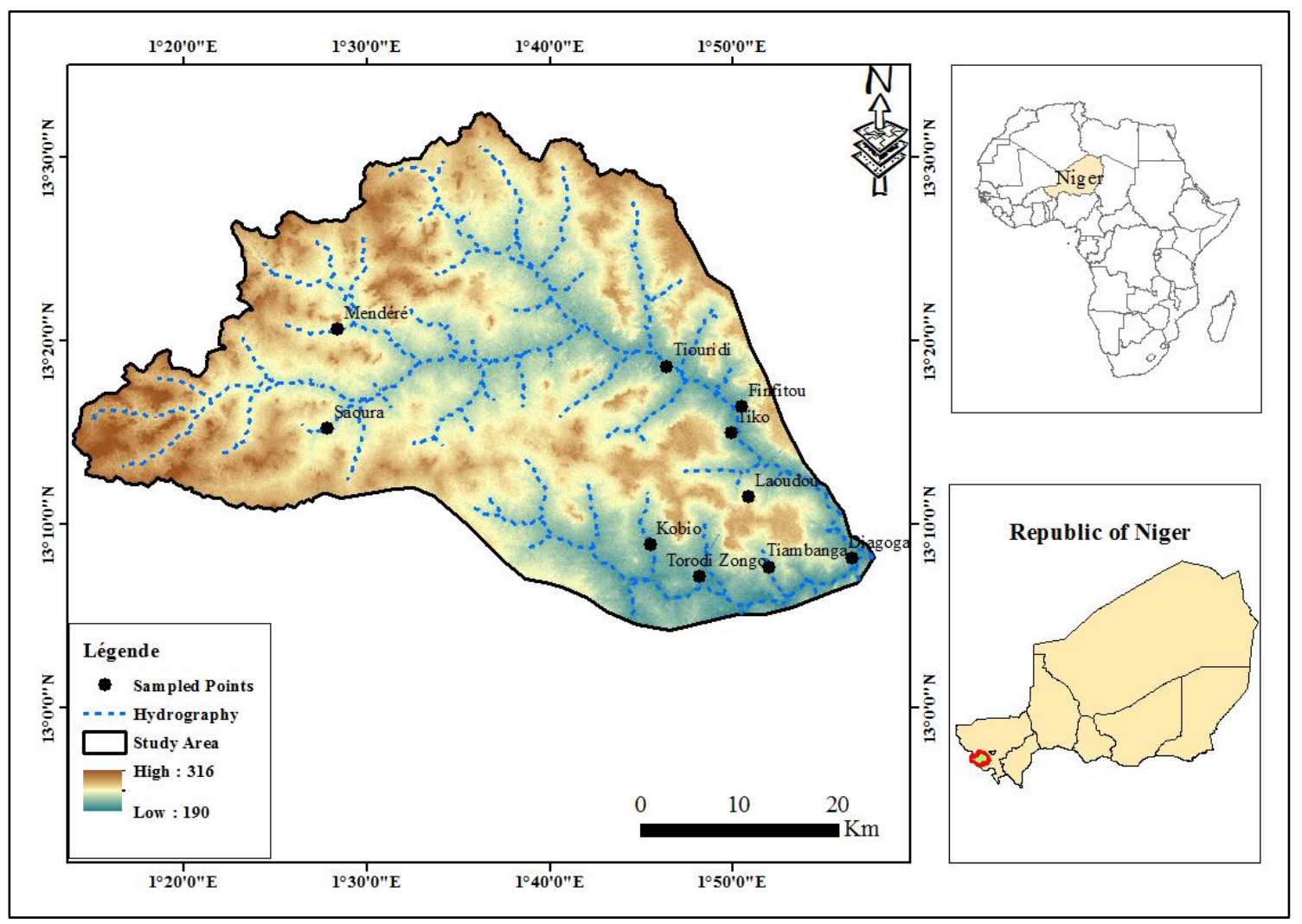

Figure 1 : Situation géographique de la zone d'étude avec les principaux points échantillonnés. 


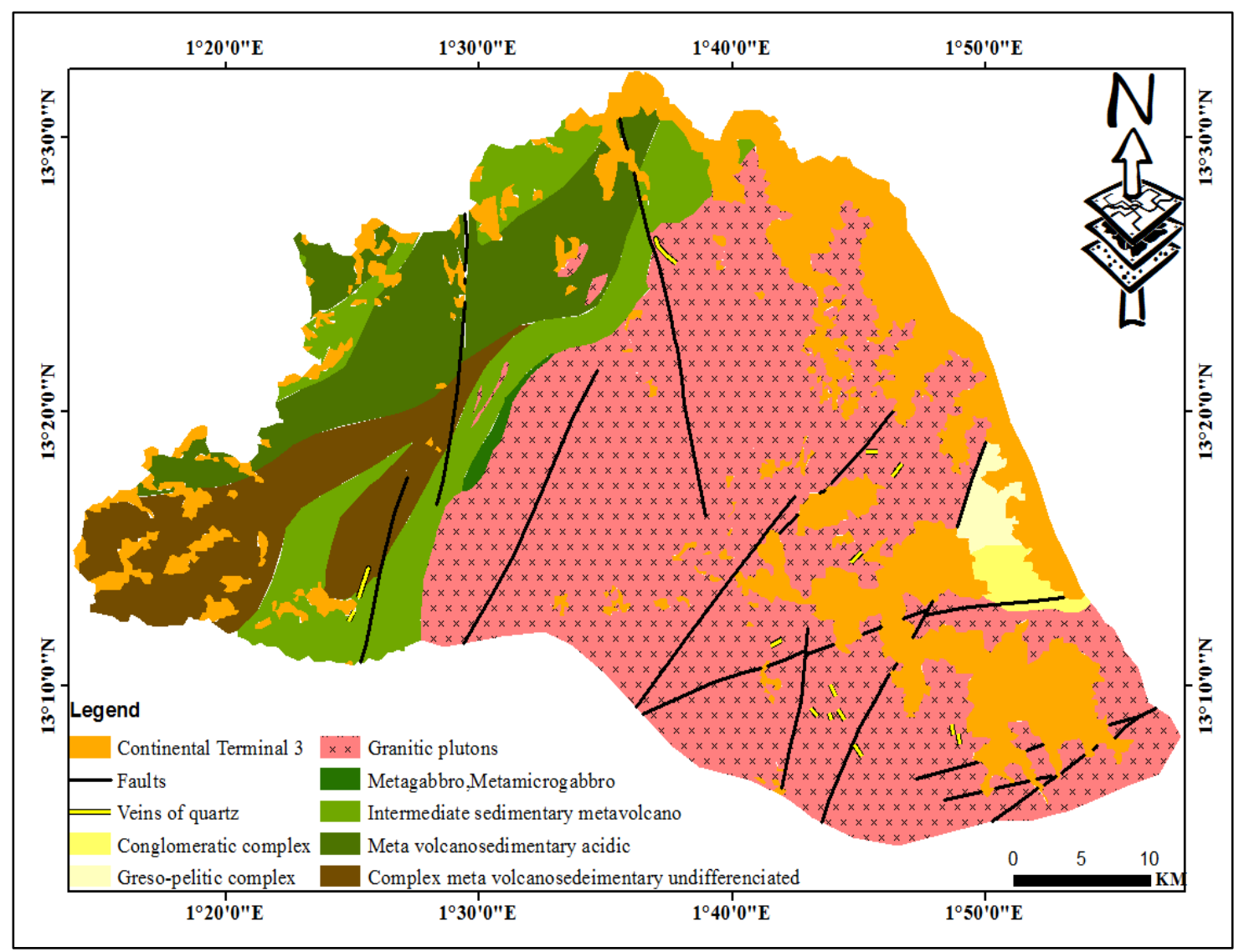

Figure 2: Carte géologique simplifiée de la zone d'étude au (1/200000) (Bonnot et al., 1998).

\section{RESULTATS}

Caractéristiques physico-chimiques des eaux et aptitude à la consommation humaine

Les résultats des analyses sont consignés dans les Tableaux (1 et 2 ). La balance ionique calculée pour l'ensemble des échantillons d'eau était $\leq \pm 6 \%$. Ce qui a permis de prendre en compte de tous les résultats d'analyses.

\section{Mesures in situ}

Ces résultats ont révélé des valeurs des températures variant entre 28,5 et $34,1^{\circ} \mathrm{C}$ pour une moyenne de $31,25^{\circ} \mathrm{C}$ et un écart type de $1,94(\mathrm{n}=10)$. Le $\mathrm{pH}$ des eaux est généralement compris entre 5,91 et 7,5 unités $\mathrm{pH}$ avec une moyenne de 6,9 et un écart-type de 0,57 caractérisant ainsi des eaux acides à moyennement neutres. Les valeurs de conductivités varient de 81,1 à $557 \mu \mathrm{S} / \mathrm{cm}$ avec une moyenne de $313,57 \mu \mathrm{S} / \mathrm{cm}$, un écart type de 175,12 , montrant une faible minéralisation des eaux.

\section{Teneurs en éléments chimiques majeurs et représentations graphiques}

L'ordre de prédominance des concentrations en ions exprimés en méq/L dans les eaux analysées (Tableau 2) était respectivement donné comme suit: $\mathrm{HCO}_{3}{ }^{-}>$ $\mathrm{NO}_{3^{-}}>\mathrm{SO}_{4}{ }^{2-}>\mathrm{Cl}^{-}$pour les anions et pour les cations, se distinguait deux dispositions $\mathrm{Ca}^{2+}>$ $\mathrm{Mg}^{2+}>\mathrm{Na}^{+}>\mathrm{K}^{+}$et $\mathrm{Na}^{+}>\mathrm{Ca}^{2+}>\mathrm{K}^{+}>\mathrm{Mg}^{2+}$ montrant ainsi deux types de faciès chimiques : bicarbonaté calcique et magnésien d'une part et bicarbonaté sodique et potassique d'autre part.

Ces deux types de faciès chimiques sont corroborés par la projection des résultats 
d'analyses physico-chimiques dans le diagramme de Piper (Figure 3) qui révèle de même deux types de faciès: le faciès bicarbonaté calcique et magnésien (80\%) et le faciès bicarbonaté sodique et potassique (20\%). Quant à la projection des variables dans le diagramme de Schoeler Berkaloff (Figure 4), on constate deux familles des courbes qui sont parallèles entre elles indiquant deux familles ou deux processus de minéralisation auxquels les eaux de la zone sont soumises.

\section{Aptitude des eaux à la consommation humaine}

L'eau potable se définit comme une eau saine et exempte de toutes impuretés sans risque pour la santé humaine une fois qu'elle est consommée. Au vu de cette définition, cette étude a permis de comparer des paramètres physico-chimiques des eaux des différents forages et puits aux normes de potabilité fixées par l'OMS (2011).

Les eaux de la zone ont présenté des valeurs de $\mathrm{pH}$ variant de 5,91 à 7,5 pour une moyenne de 6,9 et de conductivités allant de 81,1 à $557 \mu \mathrm{S} / \mathrm{cm}$ avec une moyenne de 313,57 qui répondaient respectivement aux normes $(6,5-8,5)$ et $(180-1000 \mu \mathrm{S} / \mathrm{cm})$ à l'exception des eaux de Laoudou, Menderé et Saoura qui ont présenté des valeurs de $\mathrm{pH}$ légèrement acides et des faibles valeurs de conductivités. Elles ont présenté aussi des températures comprises entre 28,5 et $34,1^{\circ} \mathrm{C}$ avec une moyenne de $31,01^{\circ} \mathrm{C}$. Du point de vue cations majeurs, aucun cation n'a excédé la norme de consommation admise. Quant aux anions ce sont surtout les ions nitrates $\mathrm{NO}_{3}{ }^{-}$, dont $30 \%$ des points d'eau ont révélés des teneurs supérieures aux normes de potabilité acceptées par l'OMS $(50 \mathrm{mg} / \mathrm{l})$ et $30 \%$ des points d'eau qui avoisinaient la valeur limite. Cependant, pour les éléments en trace tels que le fer total Fet, $20 \%$ des points d'eau présentaient des valeurs supérieures à la norme de $0,3 \mathrm{mg} / \mathrm{l}$. Bien qu'indispensable à la croissance des cellules de l'organisme, à des fortes teneurs, le fer entache le linge et procure un caractère organoleptique aux eaux. Il peut aussi provoquer des maladies cardiovasculaires et à la limite des risques de cancer hépatique. La dureté de ces eaux variait de 0,05 à $1,41^{\circ} \mathrm{F}$ caractérisant des eaux très douces. Pour ce qui est de cette dernière, aucune recommandation n'a été faite par l'OMS, mais une eau très douce corrode les canalisations métalliques.

\section{Processus hydrogéochimiques Analyse en Composante Principale}

Les résultats de l'ACP sont consignés dans le Tableau 3. Douze paramètres physicochimiques $\left(\mathrm{pH}, \mathrm{T}^{\circ}, \mathrm{CE}, \mathrm{HCO}_{3}, \mathrm{SO}_{4}, \mathrm{Cl}, \mathrm{NO}_{3}\right.$, $\mathrm{Na}, \mathrm{K}, \mathrm{Mg}, \mathrm{Ca}$ et Fet) ont été pris en compte pour l'ACP. Dans la matrice de corrélation entre les différents éléments des eaux de la zone (Tableau 3) on remarque que :

$\checkmark \quad$ la Conductivité électrique $\mathrm{CE}$ a été très fortement corrélée $(>78)$ aux $\mathrm{HCO}_{3-}$ $(0,97), \mathrm{Ca}^{2}+(0,93), \mathrm{Mg} 2+$ et le $\mathrm{pH}(0,78)$. Elle a été moyennement corrélée aux $\mathrm{Na}^{+}$ $(0,70)$, corrélée négativement aux Fet ($0,64)$ et aux $\mathrm{NO}_{3}^{-}(0,65)$, expliquant les conditions dans lesquels la minéralisation est acquise et leur participation dans l'acquisition de la minéralisation des eaux de la zone ;

$\checkmark$ la température n'a pas été significativement corrélée avec le $\mathrm{Mg}$ $(0,44)$, mais a été anté-corrélée avec le FeT $(-0,72)$;

$\checkmark \quad$ le FeT est anté-corrélé avec presque tous les ions

$\checkmark \quad$ le $\mathrm{HCO}_{3}$ est très significativement corrélé avec le $\mathrm{Ca}^{2+}(0,93)$ et le $\mathrm{Mg}^{2+}(0,89)$. Il est significativement corrélé avec le $\mathrm{Na}^{+}$ $(0,62)$ et anté-corrélé avec le FeT $(-0,66)$. Ceci confirme les faciès bicarbonaté calcique à magnésien et bicarbonaté sodique des eaux, également corroborés par les diagrammes de Piper et de Schoeler Berkaloff ;

$\checkmark \quad$ le $\mathrm{Cl}$ a été significativement corrélé avec le $\mathrm{K}(0,53)$, indiquant que les deux ions pourraient provenir d'une même origine (dissolution de la sylvite ou apports 
atmosphériques à travers l'infiltration des eaux de pluies).

Cependant, Les éléments tels que $\mathrm{K}, \mathrm{Cl}$, et $\mathrm{SO}_{4}$ n'ont pas présenté des corrélations significatives avec les autres variables, telles que la Conductivité électrique C.E, donc ne jouaient pas un grand rôle dans la minéralisation des eaux de la zone.

L'analyse factorielle des variables a montré que seuls les facteurs F1, F2 et F3 présentaient des valeurs propres $>1$ avec respectivement des pourcentages de variance exprimés à $51,44 \% ; 18,19 \%$ et $12,19 \%$ (Tableau 4). Pour une somme totale de variance exprimée à $81,82 \%$, ces trois facteurs ont été considérés comme représentatifs de l'ensemble des échantillons et ont permis d'obtenir l'essentiel des informations recherchées.

Ainsi l'axe F1 représente la composante principale de minéralisation de l'eau avec $51,44 \%$ et regroupe deux types d'ions (Figure 5) qui sont :

> $\mathrm{Mg}-\mathrm{Ca}-\mathrm{HCO} 3 \mathrm{~d}$ 'une part, caractérisant les variables de l'hydrolyse des silicates et carbonates ;

$\mathrm{NO}_{3}$ - Na d'autre part caractérisant les indicateurs de pollution anthropique à travers les nitrates. Les ions $\left(\mathrm{Fe}^{2+}\right.$ et $\mathrm{Fe}^{3+}$ ) se retrouvent quant à eux au pôle négatif de l'axe F1. L'axe F1 traduit à la fois l'axe de minéralisation d'origine naturelle (contact eau-encaissant) et anthropique.
Pour ce qui est de l'axe F2 $(18,19 \%)$, il regroupe au pôle positif les ions $\mathrm{SO}_{4}$ et au pôle négatif les ions $\mathrm{K}-\mathrm{Cl}$ caractérisant les éléments issus des apports atmosphériques à travers l'infiltration des eaux de pluies. La projection des éléments dans le plan F1- F3, précise davantage les sources de minéralisation déjà identifiées dans le plan factoriel F1 - F2.

$\mathrm{La}$ projection des individus dans l'espace des unités statistiques F1-F2 scinde les différents échantillons en deux groupes d'eau et ce en fonction de la minéralisation et du $\mathrm{pH}$ (Figure 6). Les eaux faiblement minéralisées (Tiambanga, Laoudou, Mendéré et Saoura) se regroupent au pôle négatif de l'axe F1 et ceux fortement minéralisées au pôle opposé.

\section{Indice de saturation}

La forte concentration des ions $\mathrm{HCO}_{3}{ }^{-}$, $\mathrm{Ca}^{2+}$ et $\mathrm{Mg}^{2+}$ dans les eaux indiquent que ces derniers pourraient provenir de la dissolution des carbonates tels que la calcite ou la dolomite. Ce qui a conduit au calcul de l'Indice de saturation par rapport à ces deux minéraux. Les valeurs de l'indice de saturation moyen par rapport aux deux minéraux, sont respectivement plus sous-saturés pour les altérites $(-6,38$ et -12,59) que pour les aquifères du socle $(-2,45$ et 4,61$)$, et présentent cependant une certaine variation entre les aquifères de la zone (Tableau 5).

Tableau 1 : Résultats des paramètres mesurés in situ en comparaison aux normes OMS.

\begin{tabular}{ccccccc}
\hline Paramètres & unités & Normes(OMS) & Max & Min & Moy & Ecart-type \\
\hline $\mathbf{p H}$ & - & $6,5-8,5$ & 7,5 & 5,91 & 6,69 & 0,57 \\
\hline $\mathbf{C E}$ & $\mu \mathrm{s} / \mathrm{cm}$ & $180-1000$ & 557 & 81,1 & 313,57 & 175,12 \\
\hline $\mathbf{T}^{\circ}$ & ${ }^{\circ} \mathrm{C}$ & - & 34,1 & 28,5 & 31,07 & 1,94 \\
\hline
\end{tabular}


Tableau 2 : Résultats d'analyses physico-chimiques exprimés en meq/L.

\begin{tabular}{|c|c|c|c|c|c|c|c|c|c|c|c|c|}
\hline Nom & Ouvrage & Long & Lat & HCO3 & $\mathrm{Cl}$ & SO4 & $\mathbf{F}$ & NO3 & $\mathbf{N a}$ & $\mathbf{K}$ & Mg & $\mathbf{C a}$ \\
\hline Diagoga & $\mathrm{F}$ & $01^{\circ} 56^{\prime} 29^{\prime}$ & $13^{\circ} 08^{\prime} 7,5^{\prime \prime}$ & 3,7 & 0,11 & 0,17 & 0,02 & 0,86 & 2,12 & 0,15 & 1,22 & 1,6 \\
\hline Laoudou & F & $\begin{array}{l}01^{\circ} 50^{\prime} 51, \\
1^{\prime \prime}\end{array}$ & $13^{\circ} 11^{\prime 2} 27^{\prime \prime}$ & 0,5 & 0,04 & 0,04 & 0,01 & 0,14 & 0,47 & 0,07 & 0,06 & 0,14 \\
\hline Mendéré & $\mathrm{P}$ & $\begin{array}{l}01^{\circ} 28^{\prime} 22, \\
7^{\prime \prime}\end{array}$ & $13^{\circ} 20^{\prime} 35,9^{\prime}$ & 0,41 & 0,15 & 0,17 & 0,00 & 0,00 & 0,22 & 0,15 & 0,10 & 0,3 \\
\hline Saoura & $\mathrm{P}$ & $\begin{array}{l}01^{\circ} 27^{\prime} 51, \\
5^{\prime \prime}\end{array}$ & $13^{\circ} 15^{\prime} 13,1^{\prime}$ & 0,58 & 0,06 & 0,00 & 0,00 & 0,11 & 0,48 & 0,09 & 0,10 & 0,10 \\
\hline Finfitou & $\mathrm{F}$ & $\begin{array}{l}01^{\circ} 50 ' 30, \\
3^{\prime \prime}\end{array}$ & $13^{\circ} 16^{\prime} 23^{\prime \prime}$ & 3,7 & 0,03 & 0,08 & 0,01 & 0,58 & 0,42 & 0,15 & 2,33 & 1,70 \\
\hline $\begin{array}{l}\text { Tiamban } \\
\text { ga }\end{array}$ & $\mathrm{F}$ & $\begin{array}{l}01^{\circ} 51^{\prime} 59, \\
8^{\prime \prime}\end{array}$ & $13^{\circ} 07^{\prime} 38,2^{\prime}$ & 2,4 & 0,01 & 0,06 & 0,01 & 0,38 & 0,57 & 0,06 & 1,06 & 1,45 \\
\hline Tiko & $\mathrm{F}$ & $\begin{array}{l}01^{\circ} 49^{\prime} 53, \\
5^{\prime \prime}\end{array}$ & $13^{\circ} 14^{\prime} 56,2^{\prime}$ & 5,7 & 0,01 & 0,08 & 0,01 & 0,43 & 1,43 & 0,10 & 2,43 & 1,90 \\
\hline Tiouridi & $\mathrm{F}$ & $01^{\circ} 46^{\prime} 24^{\prime}$ & $13^{\circ} 18 ' 35,3^{\prime}$ & 3,10 & 0,03 & 0,38 & 0,01 & 0,13 & 0,50 & 0,05 & 1,77 & 1,55 \\
\hline Torodi Z & $\mathrm{F}$ & $01^{\circ} 48^{\prime} 9^{\prime \prime}$ & $13^{\circ} 07^{\prime} 6,9^{\prime \prime}$ & 3,00 & 0,13 & 0,08 & 0,03 & 1,02 & 0,65 & 0,09 & 2,37 & 1,47 \\
\hline zongo & $\mathrm{F}$ & $\begin{array}{l}01^{\circ} 48^{\prime} 49 \\
,,\end{array}$ & $13^{\circ} 07^{\prime} 24^{\prime \prime}$ & 3,40 & 0,42 & 0,10 & 0,02 & 0,94 & 1,52 & 0,15 & 1,82 & 1,20 \\
\hline
\end{tabular}

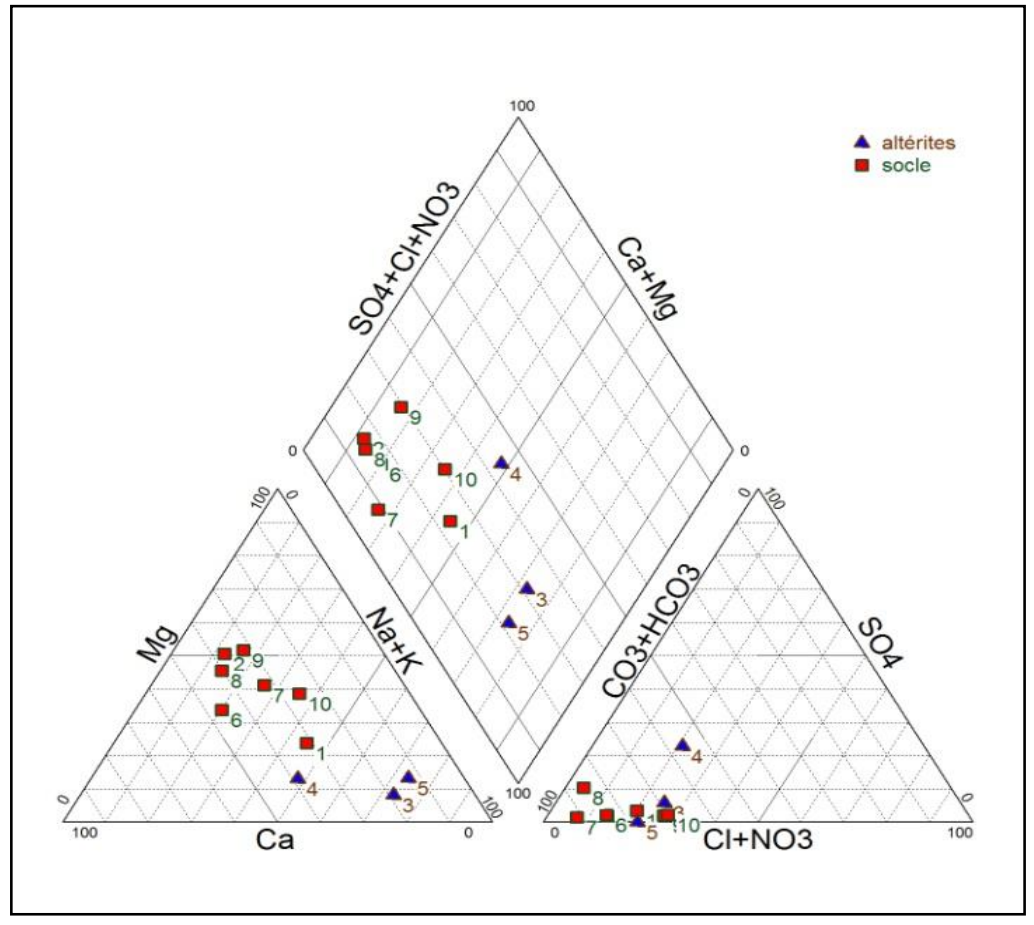

Figure 3 : Diagramme de Piper pour différentes eaux. 


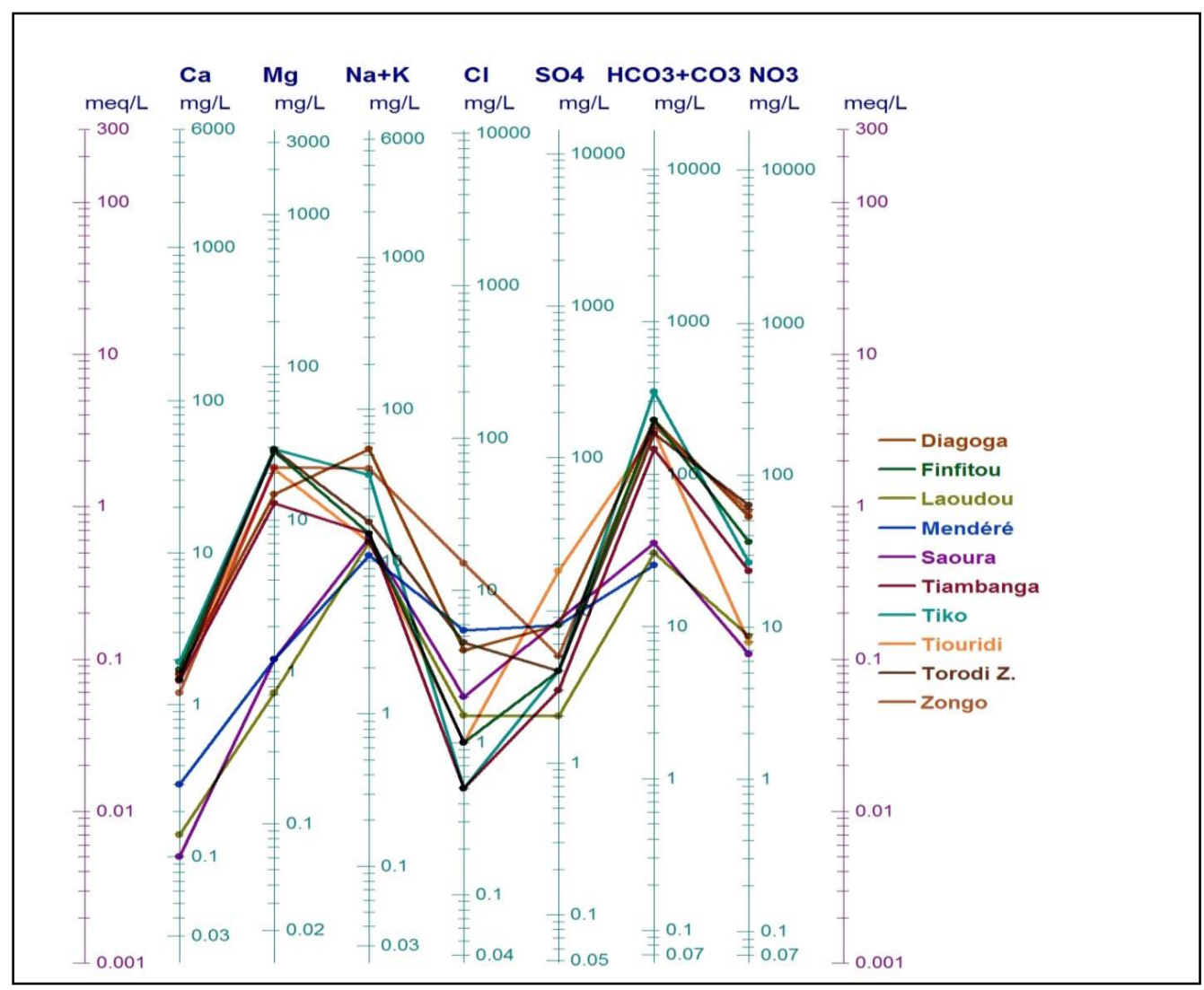

Figure 4 : Différentes familles d'eau à partir du Diagramme de Schoeler Berkaloff.

Tableau 3: Matrice de corrélation des différents ions.

\begin{tabular}{|c|c|c|c|c|c|c|c|c|c|c|c|c|}
\hline & pH & $\mathbf{T}$ & CE & $\mathbf{M g}^{2+}$ & $\mathbf{N a}^{+}$ & FeT & $\mathbf{K}^{+}$ & $\mathrm{Ca}^{2+}$ & $\mathrm{HCO}_{3}{ }^{-}$ & $\mathrm{Cl}^{-}$ & $\mathrm{SO}_{4}{ }^{2-}$ & $\mathrm{NO}_{3}{ }^{-}$ \\
\hline pH & 1 & 0,32 & 0,78 & 0,85 & 0,37 & $-0,77$ & $-0,02$ & 0,89 & 0,82 & 0,05 & 0,52 & 0,49 \\
\hline $\mathbf{T}$ & & 1 & 0,27 & 0,44 & 0,07 & $-0,72$ & $-0,3$ & 0,28 & 0,34 & $-0,11$ & $-0,19$ & 0,33 \\
\hline CE & & & 1 & 0,84 & 0,7 & $-0,64$ & 0,27 & 0,93 & 0,97 & $-0,01$ & 0,16 & 0,65 \\
\hline $\mathbf{M g}^{2+}$ & & & & 1 & 0,34 & $-0,66$ & 0,09 & 0,9 & 0,89 & 0,06 & 0,21 & 0,65 \\
\hline $\mathbf{N a}^{+}$ & & & & & 1 & $-0,43$ & 0,38 & 0,47 & 0,62 & 0,37 & 0,04 & 0,63 \\
\hline FeT & & & & & & 1 & 0,29 & $-0,7$ & $-0,66$ & 0,07 & $-0,11$ & $-0,56$ \\
\hline $\mathbf{K}^{+}$ & & & & & & & 1 & 0,06 & 0,16 & 0,53 & $-0,09$ & 0,35 \\
\hline $\mathrm{Ca}^{2+}$ & & & & & & & & 1 & 0,93 & $-0,09$ & 0,33 & 0,58 \\
\hline $\mathrm{HCO}_{3}^{-}$ & & & & & & & & & 1 & 0 & 0,2 & 0,58 \\
\hline Cl- & & & & & & & & & & 1 & $-0,01$ & 0,49 \\
\hline $\mathrm{SO}_{4}{ }^{2-}$ & & & & & & & & & & & 1 & $-0,13$ \\
\hline $\mathrm{NO}_{3}{ }^{-}$ & & & & & & & & & & & & 1 \\
\hline
\end{tabular}


Tableau 4: Valeurs propres et pourcentage de variance exprimée.

\begin{tabular}{llll}
\hline & F1 & F2 & F3 \\
\hline Valeur propre & 6,17 & 2,18 & 1,46 \\
Total variance (\%) & 51,43 & 18,19 & 12,19 \\
Cumul\% & 51,43 & 69,62 & 81,82 \\
\hline
\end{tabular}
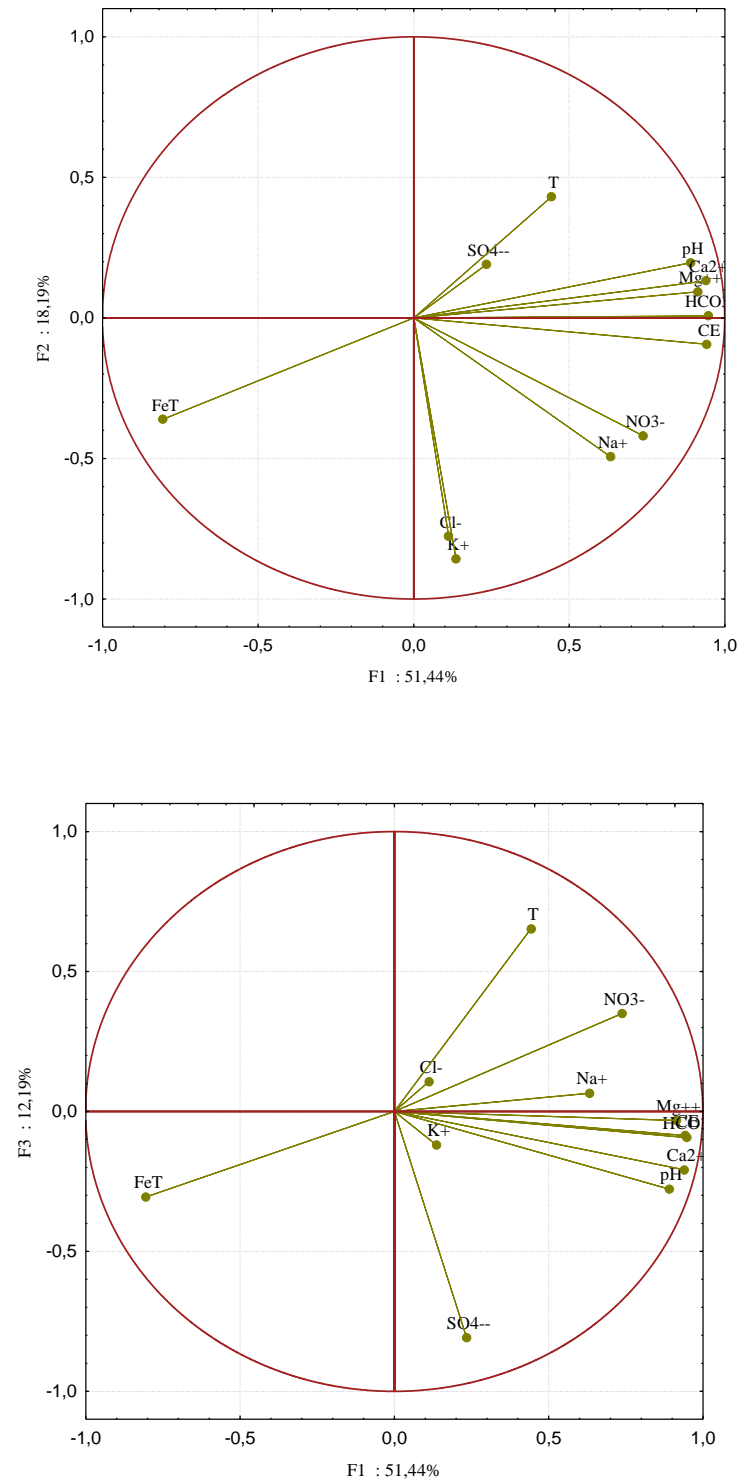

Figure 5: Projections des variables dans le plan factoriel (F1-F2) et (F1-F3). 


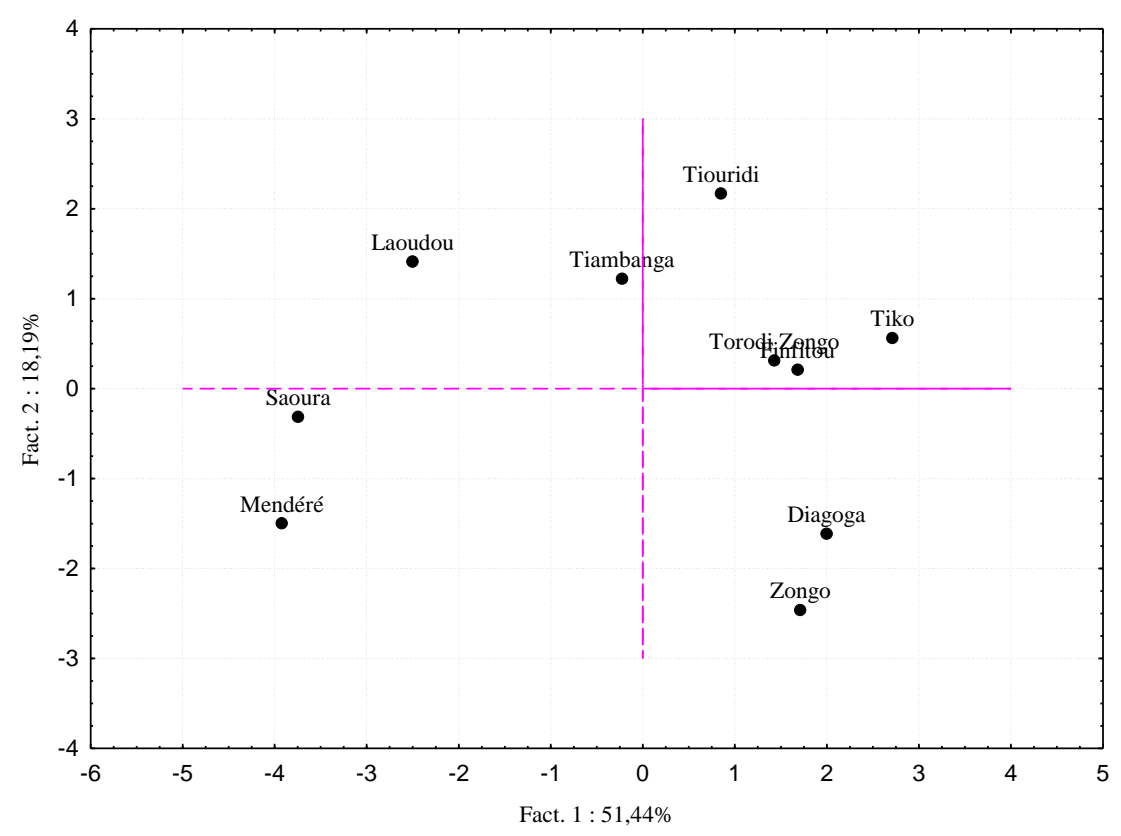

Figure 6: Projection des individus dans le plan (F1-F2).

Tableau 5: Valeurs des Indices de saturation vis-à-vis de la Calcite, de la dolomite, et du Gypse.

\begin{tabular}{lllll}
\hline & & Is Calcite & \multicolumn{2}{c}{ Is Dolomite } \\
\hline & Altérites $(\mathbf{n}=\mathbf{3})$ & Socle $(\mathbf{n}=7)$ & Altérites & Socle \\
\hline Moyenne & $-6,38$ & $-2,45$ & $-12,59$ & 4,61 \\
Max & $-6,17$ & $-3,1$ & $-12,47$ & $-5,8$ \\
Min & $-6,53$ & $-3,95$ & $-12,7$ & $-7,69$ \\
\hline
\end{tabular}

\section{DISCUSSION}

Les résultats d'analyses physicochimiques ont montré que les eaux de la zone présentaient des valeurs de température variant de 28,5 à $34,1^{\circ} \mathrm{C}$. Ces dernières sont proches des valeurs obtenues dans d'autres régions du socle du Niger notamment dans le Sud Zinder et dans le Nord-Ouest du Liptako (Abdou Babayé et al., 2016 ; Souley et al., 2018). Elles reflètent les valeurs de températures atmosphériques moyennes observées dans les régions arides (Abdou Babayé, 2012 ; Hassane Saley, 2018). Le pH des eaux étaient légèrement acides (forages de Diagoga, Laoudou, Tiambanga, Torodi Zongo et les puits de Saoura et Mendéré) à moyennement neutres (forages de Zongo, Tiko, Tiouridi et Finfitou). Cette acidité a été d'autant plus observée au niveau des ouvrages captant les aquifères superficiels notamment les alluvions dont la nature géologique du réservoir est principalement constituée par des formations sablo-argileuses. Pour les aquifères profonds, la nature silicatée des réservoirs en système ouvert et la présence du $\mathrm{CO}_{2}$ du sol, maintiennent le $\mathrm{pH}$ acide. L'ordre de prédominance des anions $\mathrm{HCO}_{3}-\mathrm{NO}_{3}-\mathrm{SO}_{4}-\mathrm{Cl}$ et pour les cations $\mathrm{Ca}-\mathrm{Na}-\mathrm{Mg}-\mathrm{K}$ est le même que celui observé par Babayé et al. (2016) dans des zones un peu similaires avec cependant quelques différences pour les anions où les ions $\mathrm{Cl}$ et $\mathrm{SO}_{4}$ dominent sur les ions $\mathrm{NO}_{3}$. Deux 
types de faciès ont été relevés: le faciès bicarbonaté sodique et potassique (20\%) et le faciès bicarbonaté calcique et magnésien plus dominant (80\%). Ce faciès bicarbonaté dominant était surtout caractéristique des zones de socle de l'Afrique de l'Ouest en général (Kouassi et al., 2010; Lasm et al., 2011; Ayouba et Guel, 2015) et celles des régions de socle du Niger en particulier (Amadou et al., 2014 ; Abdou Babayé et al., 2016 ; Souley et al., 2018). La prédominance des ions $\mathrm{HCO}_{3}$ à l'origine de ce faciès s'explique par l'hydrolyse des silicates en présence du $\mathrm{CO}_{2}$ du sol et/ou la dissolution des carbonates.

Les eaux souterraines acquièrent généralement leur minéralisation soit à partir des interactions eau-roche à travers les différents échanges cationiques pouvant s'établir, soit à partir des apports d'origine superficielle ou anthropique. Ces interactions se traduisent par l'altération des minéraux primaires des roches silicatées par hydrolyse ou par dissolution. Aussi, au contact des eaux riches en $\mathrm{CO}_{2}$, les minéraux silicatés s'altèrent en donnant ainsi des argiles de néoformation tout en libérant les cations les plus mobiles tels que $\mathrm{Na}^{+}, \mathrm{K}^{+}, \mathrm{Ca}^{2+}$ et $\mathrm{Mg}^{2+}$ (Babayé, 2012). Les différentes corrélations observées entre divers éléments dans la matrice de corrélation témoignent de leur origine. En effet, ce sont les ions $\mathrm{HCO}_{3}, \mathrm{Mg}, \mathrm{Na}, \mathrm{Ca}$ et $\mathrm{NO}_{3}$ qui contrôlent la minéralisation des eaux de la zone. Les valeurs des indice de saturation, Les fortes corrélations entre $\mathrm{HCO}_{3}$ avec les ions $\mathrm{Ca}(0,93)$ et $\mathrm{Mg}(0,90)$ ainsi que leur regroupement autour de l'axe principal de minéralisation F1, indiquent qu'ils proviennent probablement d'un même mécanisme notamment de l'hydrolyse des silicates ferromagnésiens de la zone tels que les amphiboles et la biotite traduisant le temps de séjour de l'eau dans le réservoir. Le calcul des indices de saturations par rapport aux minéraux carbonatés que sont la calcite, la dolomite a montré une sous saturation des eaux de par rapport à ces minéraux, cette sous saturation traduit une éventuelle dissolution de ces minéraux contribuant aussi à l'acquisition de la minéralisation dans les roches réservoir. Les réactions de mise en solution de ces ions sont les suivantes :

$\mathrm{KMg}_{3} \mathrm{AlSi}_{3} \mathrm{O}_{10}(\mathrm{OH})_{2}(\mathrm{Phlogopite})+\mathbf{7 H}_{2} \mathrm{CO}_{3}$ $+0,5 \mathrm{H}_{2} \mathrm{O} \rightarrow \mathrm{K}^{+}+3 \mathrm{Mg}^{2+}+2 \mathrm{H}_{4} \mathrm{SIO}_{4}$ (Silice) + $0,5 \mathrm{Si}_{2} \mathrm{O}_{3}(\mathrm{OH})_{4} \mathrm{Al}_{2}$ (Kaolinite) (4)

$\mathrm{Si}_{2} \mathrm{Al}_{2} \mathrm{O}_{8} \mathrm{Ca}$ (Anorthite) $+2 \mathrm{CO}_{2}+3 \mathrm{H}_{2} \mathrm{O} \rightarrow$ $\mathrm{Si}_{2} \mathrm{O}_{3}(\mathrm{OH})_{4} \mathrm{Al}_{2}$ (Kaolinite) $+2 \mathrm{HCO}_{3}+\mathrm{Ca}^{2+}$ (5)

Le calcul des indices de saturations par rapport aux minéraux carbonatés que sont la calcite, la dolomite a montré une sous saturation des eaux de par rapport à ces minéraux, cette sous saturation traduit une éventuelle dissolution de ces minéraux contribuant aussi à l'acquisition de la minéralisation des eaux de la région.

Les ions $\mathrm{NO}_{3}-\mathrm{Na}$ bien qu'aussi regroupés autour de l'axe F1 comme les ions $\mathrm{HCO}_{3}-\mathrm{Ca}-\mathrm{Mg}$, traduisent quant à eux un mécanisme de minéralisation différent de l'hydrolyse. En effet, les nitrates $\mathrm{NO}_{3}{ }^{-}$ainsi que le sodium $\mathrm{Na}^{+}$sont souvent d'origine superficielle, car la concentration naturelle des nitrates dans les eaux souterraines en l'absence de fertilisation ne peut dépasser les $15 \mathrm{mg} / \mathrm{l}$. Ces ions se retrouvent aussi dans les eaux suite aux activités anthropiques notamment par les activités agricoles et pastorales à travers l'épandage des engrais ainsi que par la concentration des déchets humains et animaux en relation avec la nature moins profonde de ces ouvrages. Le Fer $\mathrm{Fe}$ se retrouve sous forme ferrique ou ferreux dans les eaux à partir de la décomposition des roches riches en ions ferromagnésiens tels que les oxydes de fer. Sa corrélation avec la température confirme son origine naturelle. Les ainsi les deux mécanismes contrôlant la minéralisation des eaux de cette zone était l'hydrolyse des silicates et les apports anthropiques et dans une certaine mesure la dissolution des minéraux carbonatés.

\section{Conclusion}

La combinaison des méthodes hydrochimiques aux études statistiques basées sur l'ACP ainsi qu'au calcul de l'indice de saturation a permis de caractériser 
chimiquement les aquifères du socle du moyen bassin versant de Goroubi. Avec des valeurs de $\mathrm{pH}$ variant de 5,91-7,5 unités $\mathrm{pH}$; de faibles valeurs de conductivités, ces eaux étaient légèrement acides et faiblement minéralisées. Cette faible minéralisation a été surtout confirmée par le calcul de l'indice de saturation en rapport avec la nature lithologique des réservoirs. Aussi, les fortes teneurs en fer et en nitrates des eaux de certains points faisaient de ces eaux impropres à la consommation humaine. Ainsi, on a constaté que pour les dix échantillons sélectionnés sur les $28,30 \%$ sont impropres à la consommation humaine tandis que $20 \%$ sont indésirables. Enfin, les résultats de l'ACP, ont indiqué deux facteurs contrôlant le processus de cette minéralisation dans cette zone à savoir l'hydrolyse des silicates ainsi qu'une pollution d'origine anthropique par les nitrates. Ainsi, les principaux ions sont en majorité issus de l'hydrolyse des silicates, et des apports anthropiques.

\section{CONFLIT D'INTERETS}

Les auteurs déclarent qu'ils n'ont aucun conflit d'intérêts.

\section{CONTRIBUTIONS DES AUTEURS}

DSD est l'investigateur général ayant collecté, analysé et rédigé le manuscrit. $\mathrm{OB}$ a contribué à l'interprétation des résultats et à la rédaction du manuscrit. SI, AY et $\mathrm{MH}$ ont contribué à la lecture et à la correction du manuscrit.

\section{REMERCIEMENTS}

Nous adressons nos sincères remerciements à la Direction Régionale de l'Hydraulique et de l'Assainissement de Tillabéri, Niger pour leur contribution aux analyses des échantillons.

\section{REFERENCES}

Hassane-Saley AK. 2018. Évaluation des ressources en eau de l'aquifère du Continental Intercalaire/Hamadien de la région de Tahoua (bassin des Iullemmeden, Niger): impacts climatiques et anthropiques. Thèse de Doctorat, Université Paris-Sud et Université Abdou Moumouni de NiameyNiger, 295p.

Hassane-Saley AK, Sandao I, Michelot JL, Ousmane B. 2019. Apport des paramètres physico-chimiques des eaux à l'amélioration de la connaissance des aquifères $\quad \mathrm{du}$ Continental Intercalaire/Continental Hamadien de la région de Tahoua (Bassin des Iullemmeden, Niger). European Scientific Journal, 15(12): $444 . \quad$ DOI: http//doi.org/10.19044/esj.2019.v15n12p 444

Ayouba AM, Guel B. 2015. Caractérisation physico-chimique des eaux souterraines de la localité de Yamtenga (Burkina Faso) Int. J. Biol. Chem. Sci., 9(1): 517-533. DOI: http//dx.doi ;org/10.4314/ijbcs.v9i.44

Ousmane B, Djibo S, Soumana I, Soussou A. 2010. Étude préliminaire de la pollution bactériologique des eaux des aquifères discontinus du socle du département de Téra /Liptako nigérien. Afrique Science, 06(3): 27 - 36

Favreau G, Cappelaere B, Massuel S, Leblanc M, Boucher M, Boulain N, Leduc C. 2009. Land clearing, climate variability, and water resources increase in semiarid southwest Niger: A review. Water Resour. Res., $\quad 45: \quad 1-18 . \quad$ DOI: 10.1029/2007WR006785

Sandao I, Hassane SAK, Haladou MBG, Moussa MM, Ousmane B. 2019. Teneur en Fluor dans les eaux souterraines et ses impacts dans l'alimentation en eau des populations du Nord-Ouest de la Région de Zinder, Niger. Afrique Science, 15(5) : $340-351$.

Abdou Babayé MS. 2012. Evaluation des ressources en eau souterraine dans le bassin de Dargol (Liptako-Niger). Thèse de Doctorat, Université de LiègeBelgique et Université Abdou Moumouni de Niamey-Niger, 265p. 
Amadou H, Laouali MS, Manzola A. 2014 Application des méthodes d'analyses statistiques multi variées à l'étude de la minéralisation des eaux de la zone de Zinder (Sud-Est du Niger). Int. J. Biol. Chem. Sci., 8(4): 1904-1916. DOI: http://dx.doi.org/10.4314/ijbcs.v8i4.50

Bonnot H, Abdou A, Bori Kadey D, Saint Martin M, Younfa L. 1998. Carte géologique du Niger au 1/200000, feuille de Niamey. Ministère de la Recherche Géologique Minière et Ministère français de la coopération.

Yao KT. 2009. Hydrodynamisme dans les aquifères de socle cristallin et cristallophyllien du Sud-ouest de la Côte d'Ivoire : cas du département de Soubré : apports de la télédétection, de la géomorphologie et de l'hydrogéochimie, Thèse, Université de Cocody, Cote d'Ivoire, $338 \mathrm{p}$.

Souley MR, Malam Alma MM, Mahaman SL, Natatou I, Habou I. 2018. Caractérisation physico-chimique des eaux souterraines du socle de la région de Zinder(Niger) pendant la saison des pluies et la saison sèche. European Scientific Journal, 14(27): 7857-7881. DOI: 10.19044/esj.2018.v14n27p317

Abdou Babayé MS, Sandao I, Saley MB, Wagani I, Ousmane B. 2016. Comportement hydrogéochimique et contamination des eaux des aquifères fissures du socle précambrien en milieu semi-aride (sud-ouest du Niger) Int. J. Biol. Chem. Sci., 10(6): 2728-2743. DOI : http://dx.doi.org/10.4314/ijbcs.v10i6.26

Kouassi MA, Yao AK, Ahoussi EK, Seki CL, Yao AN, Kouassi IK, Biémi J. 2010. Apports des méthodes statistiques et hydrochimiques à la caractérisation des eaux des aquifères fissurés de la région du N'zi-Comoé (Centre-Est de la Côte d'Ivoire) Int. J. Biol. Chem. Sci., 4(5): 1816-1838.

DOI: http://dx.doi.org/10.4314/ijbcs.v6i2.31

Lasm T, De Lasme O, Oga MSY, Youan Ta M, Baka D, Kouame F, Yao TK. 2011. Caractérisation hydrochimique des aquifères fissurés de la région de SanPedro (Sud-Ouest de la Côte d'Ivoire). Int. J. Biol. Chem. Sci., 5(2): 642-662. DOI: $10.4314 /$ ijbcs.v5i2.72129

Malam Salmanou S. 2018. Evaluation des ressources en eau dans la Commune rurale de Droum, haut bassin versant de la Korama/Région de Zinder. Thèse, Université Abdou Moumouni de Niamey, Niger, $193 \mathrm{p}$.

Tableau de Bord Santé-Environnement. 2007. Région Rhône-Alpes: observatoire régionale de la santé Rhône-Alpes. PRSE, Grande Bretagne.

Programme Solidarité Eau. 2018. Programme eau et assainissement pour un développement durable dans la commune rurale de Torodi. PSE, France. 